\title{
Monoclonal Antibodies Counteract Opioid-Induced Behavioral and Toxic Effects in Mice and Rats $\mathbf{9}$
}

\author{
(1) Carly Baehr, April Huseby Kelcher, Aaron Khaimraj, Dana E. Reed, Sujata G. Pandit, \\ David AuCoin, Saadyah Averick, and (1) Marco Pravetoni
}

\begin{abstract}
Departments of Pharmacology (C.B., A.H.K., A.K., M.P.), Veterinary Population Medicine (C.B.), and Psychiatry and Behavioral Sciences (A.H.K.), University of Minnesota Medical School, Minneapolis, Minnesota; Reno School of Medicine, University of Nevada, Reno, Nevada (D.E.R., S.G.P., D.A.); Allegheny Health Network, Pittsburgh, Pennsylvania (S.A.); and Center for Immunology, University of Minnesota, Minneapolis, Minnesota (M.P.)
\end{abstract}

Received May 22, 2020; accepted September 23, 2020

\begin{abstract}
Monoclonal antibodies (mAbs) and vaccines have been proposed as medical countermeasures to treat opioid use disorder (OUD) and prevent opioid overdose. In contrast to current pharmacotherapies (e.g., methadone, buprenorphine, naltrexone, and naloxone) for OUD and overdose, which target brain opioid receptors, mAbs and vaccine-generated polyclonal antibodies sequester the target opioid in the serum and reduce drug distribution to the brain. Furthermore, mAbs offer several potential clinical benefits over approved medications, such as longer serum half-life, higher selectivity, reduced side effects, and no abuse liability. Using magnetic enrichment to isolate opioid-specific B cell lymphocytes prior to fusion with myeloma partners, this study identified a series of murine hybridoma cell lines expressing mAbs with high affinity for opioids of clinical interest, including oxycodone, heroin and its active metabolites, and fentanyl. In mice, passive immunization with lead mAbs against oxycodone, heroin, and
\end{abstract}

fentanyl reduced drug-induced antinociception and the distribution of the target opioid to the brain. In mice and rats, mAb pretreatment reduced fentanyl-induced respiratory depression and bradycardia, two risk factors for opioid-related overdose fatality. Overall, these results support use of mAbs to counteract toxic effects of opioids and other chemical threats.

\section{SIGNIFICANCE STATEMENT}

The incidence of fatal overdoses due to the widespread access to heroin, prescription opioids, and fentanyl suggests that current Food and Drug Administration-approved countermeasures are not sufficient to mitigate the opioid epidemic. Monoclonal antibodies (mAbs) may provide acute protection from overdose by binding to circulating opioids in serum. Use of mAbs prophylactically, or after exposure in combination with naloxone, may reduce hospitalization and increase survival.

\section{Introduction}

An estimated 2.5 million people in the United States are living with an opioid use disorder (OUD), and 67,000 fatal drug overdoses occurred in the United States in 2018, of which $70 \%$ involved opioids (Centers for Disease Control and Prevention, 2020). Current interventions for OUD consist of

This work was supported by the National Institute on Drug Abuse, National Institute of Allergy and Infectious Diseases, and the Office of The Director of the National Institutes of Health under CounterACT Administrative Supplement to award number [U01DA038876] and under award number [U01DA051658] (to M.P.); and by the National Institutes of Health under [T32DA007097] (to C.B.) and [T32DA037183] (to A.H.K.). The content is solely the responsibility of the authors and does not necessarily represent the official views of the National Institutes of Health. Dynamic light scattering analysis was conducted in the Minnesota Nano Center, which is supported by the National Science Foundation through the National Nano Coordinated Infrastructure Network (NNCI) [Award Number ECCS-1542202]. Disclosures of interest: MP and CB are inventors of PCT application "Anti-opioid compounds and methods of making and using same." All other authors have no conflicts of interest.

https://doi.org/10.1124/jpet.120.000124.

S This article has supplemental material available at jpet.aspetjournals.org. pharmacological agonists (methadone), partial agonists (buprenorphine), and antagonists (naloxone and naltrexone) targeting the opioid receptors in the brain to exert therapeutic effects. Although opioid pharmacotherapy has substantial clinical utility in medication-assisted treatment of OUD, and naloxone is a critical emergency medication for reversing opioid overdose, these medications have been insufficient to curb the prevalence of OUD and incidence of overdose (Sharma et al., 2017; Han et al., 2019). Limitations of these medications include undesired side effects, abuse liability or diversion of agonists, the need for detoxification prior to initiation of antagonist treatment to avoid symptoms of precipitated withdrawal, and the requirement for frequent dosing, which presents a high burden of compliance. Consequently, complementary or alternative therapies are needed to supplement current medications.

Immunotherapeutics, consisting of monoclonal antibodies $(\mathrm{mAbs})$ and vaccines, offer a promising strategy to treat OUD and reduce the incidence of overdose (reviewed in Bremer and Janda (2017), Pravetoni and Comer (2019)). Monoclonal

ABBREVIATIONS: Ab, antibody; 6-AM, 6-monoacetyl morphine; BLI, biolayer interferometry; BSA, bovine serum albumin; F, fentanyl; GC-MS, gas chromatography-mass spectrometry; HRP, horseradish peroxidase; IL-4, interleukin-4; LC-MS, liquid chromatography-mass spectrometry; M, morphine; mAb, monoclonal antibody; OUD, opioid use disorder; OXY, oxycodone; PE, phycoerythrin; sKLH, subunit keyhole limpet hemocyanin. 
antibodies and vaccine-induced polyclonal antibodies selectively alter the pharmacokinetics of the target drug through binding and sequestration of drug molecules in serum, preventing drug distribution to the brain without directly affecting receptor signaling. Both $\mathrm{mAbs}$ and vaccines may offer several advantages over opioid antagonists, including fewer side effects; additionally, pharmacotherapy may require controlled detoxification to prevent precipitated withdrawal (Jarvis et al., 2018; Rzasa Lynn and Galinkin, 2018), whereas $\mathrm{mAbs}$ and vaccines are not expected to alter endogenous opioid signaling or to require detoxification (Raleigh et al., 2020). Additionally, antibodies typically exhibit high specificity for their target with little cross-reactivity for structurally distinct opioid agonists or antagonists (Raleigh et al., 2017). Therefore, mAbs and vaccines can be considered both as an alternative and as a supplement to existing small molecule therapies for OUD.

Anti-opioid vaccines have demonstrated preclinical efficacy and selectivity in reducing opioid distribution to the brain, opioid-induced respiratory depression and antinociception, intravenous self-administration, and lethality in rodent and nonhuman primate models (Pravetoni et al., 2013; Bremer et al., 2017; Raleigh et al., 2017; Nguyen et al., 2018; Sulima et al., 2018; Tenney et al., 2019). However, the efficacy of antidrug vaccines is dependent on generation of high concentrations of polyclonal antibodies, which may require multiple immunizations over weeks or months. Furthermore, active immunization may only achieve sufficient antibody concentrations in a subset of individuals (Cornuz et al., 2008; Martell et al., 2009; Kosten et al., 2014). In contrast, direct administration of high-affinity drug-specific mAbs would provide almost immediate protection against the target drug and allow for greater control over serum antibody concentrations. Drug-targeting mAbs have demonstrated preclinical efficacy against cocaine, methamphetamines, nicotine, and opioids (Fox et al., 1996; Keyler et al., 2005; Kashanian et al., 2015; Pravetoni, 2016; Kvello et al., 2019; Marckel et al., 2019; Smith et al., 2019). Additionally, favorable safety and pharmacokinetic profiles for a chimeric $\mathrm{mAb}$ against methamphetamine support the clinical translation of mAbs for OUD (Stevens et al., 2014).

Decades after its invention, hybridoma technology remains an effective method for generation of novel mAbs. However, fusion of antibody (Ab)-expressing cells with myeloma fusion partner cells is a stochastic event, and isolation of desired clones stably expressing mAbs against the antigen of interest often requires screening of hundreds of clones. To streamline the generation of hybridomas, it has been reported that antigen-based magnetic enrichment can be used to preselect target-specific B cells prior to hybridoma fusion (Spanier et al., 2016). Magnetic enrichment or "baiting" is frequently employed to increase a desired cell population for flow cytometry analysis (Boonyaratanakornkit and Taylor, 2019), and single-cell sorting has been used for isolation of antigenspecific $B$ cells and development of recombinant mAbs against a variety of targets and in multiple species (Smith et al., 2009; Ho et al., 2016; Starkie et al., 2016; Lei et al., 2019). Using an antigen-based enrichment platform previously validated for flow cytometric analysis of opioid-specific B cell populations (Taylor et al., 2014; Laudenbach et al., 2015), hybridomas were isolated from mice vaccinated against three commonly misused opioids: oxycodone, heroin, and fentanyl. The mAbs isolated using this method demonstrated binding to their target drug in vitro, as well as in vivo efficacy in reducing opioid distribution and behavioral effects when administered to mice and rats, supporting further preclinical development of opioid-targeting mAbs as a therapy to treat OUD and prevent overdose.

\section{Materials and Methods}

Animals. All procedures were approved by the Institutional Animal Care and Use Committees of the University of Minnesota and Hennepin Healthcare Research Institute and conducted in accordance with the Guide for the Care and Use of Laboratory Animals (8th Edition, National Academies Press). Male and female Balb/c mice (Jackson Laboratory, Bar Harbor, ME) were 7 weeks old on arrival, and male Sprague Dawley rats (Envigo, Indianapolis, IN) were 8-10 weeks old on arrival. All animals were housed under standard conditions with a 12/12 hour light/dark cycle and given food and water ad libitum.

Haptens and Conjugates. The oxycodone (OXY), morphine (M), and fentanyl $(\mathrm{F})$ haptens containing a tetraglycine $\left[(\mathrm{Gly})_{4}\right]$ linker were synthesized as previously described (Pravetoni et al., 2012; Raleigh et al., 2013, 2019) and then conjugated via carbodiimide chemistry (Baruffaldi et al., 2018) to the subunit keyhole limpet hemocyanin (sKLH) carrier protein for immunogens, phycoerythrin (PE) for magnetic enrichment, and either ovalbumin or bovine serum albumin (BSA) for screening assays. An additional fentanyl-based hapten ( $\mathrm{Li}$ et al., 2017) containing a biotin moiety was synthesized and used for antibody characterization by biolayer interferometry (BLI; Supplemental Fig. 6).

Active Immunization and Hybridoma Fusion. Male Balb/c mice ( $n=4$ per group) were immunized on days 0 and 28 (vaccine formulations detailed in Supplemental Table 1), and pooled lymph nodes and spleens were collected 4 days after the second immunization. The antigen-based magnetic enrichment procedure to isolate opioid-specific B cells was performed as described in Robinson et al. (2019). Briefly, tissues were processed to a single-cell suspension, and cells were pelleted at 1600 rpm for 5 minutes. Pellets were resuspended in DMEM, the opioid-based hapten conjugated to PE was added to a final concentration of $6.7 \mathrm{nM}$, and the mixture was incubated for 25 minutes at room temperature. Cells were washed with $10 \mathrm{ml}$ DMEM, and pellet was resuspended in $125 \mu \mathrm{l}$ DMEM with $25 \mu \mathrm{l}$ anti-PE microbeads (Miltenyi Biotec, Auburn, CA). Cells and beads were incubated for 15 minutes at room temperature, and antigen-specific cells collected using magnetic separator columns (Miltenyi). Columns were washed with DMEM, and antigen-specific cells were eluted with $5 \mathrm{ml}$ ClonaCell-HY Medium A (StemCell Technologies, Cambridge, MA). The enriched antigen-specific cells were counted, washed with serum-free media, and fused with Sp2/ 0Ag14 (CRL1581; American Type Culture Collection, Manassas, VA) mouse myeloma cells at a 1:5 ratio using the ClonaCell-HY Hybridoma kit (StemCell) according to the manufacturer's recommended protocol. Fused hybridoma cells were grown with hypoxanthine-aminopterinthymidine selection for $10-14$ days at $37^{\circ} \mathrm{C}$ and $5 \% \mathrm{CO}_{2}$, and visible colonies were transferred to 96 -well plates containing $100 \mu \mathrm{l}$ culture medium per well and incubated for $2-4$ days prior to screening.

Hybridoma Screening. To screen for antigen-positive colonies by ELISA, 96-well plates were coated with $5 \mathrm{ng} /$ well OXY-ovalbumin, M-BSA or F-BSA, blocked with $1 \%$ gelatin, and incubated with $50 \mu \mathrm{l}$ conditioned medium diluted 1:1 with PBS+0.05\% Tween-20 for 2 hours. Plates were washed and incubated with horseradish peroxidase (HRP)-conjugated goat antimouse secondary antibody (Jackson) overnight, and HRP activity was measured using SigmaFast OPD substrate (Millipore Sigma) with absorbance read at $492 \mathrm{~nm}$ on a Tecan Infinite M1000 microplate reader (Tecan, Männedorf, Switzerland).

Determination of Relative Antibody Affinity. For competitive ELISA, 96-well plates were coated with $5 \mathrm{ng} /$ well cognate antigen overnight and blocked with $1 \%$ gelatin. Plates were incubated with 
purified antibody, 0.04-0.06 $\mu \mathrm{g} / \mathrm{ml}$, for 2 hours in the presence of free opioid as competitor in a range of concentrations ( $1 \mathrm{mM}$ to $1 \mathrm{pM})$. Plates were washed and incubated overnight with HRP-conjugated goat antimouse secondary antibody, and HRP activity was measured using SigmaFast OPD substrate. BLI was performed using ForteBio BLItz system (Molecular Devices, San Jose, CA) with streptavidin biosensors. Biosensors were loaded with $2 \mu \mathrm{M}$ fentanyl-biotin (see Supplemental Material for synthesis) for 60 seconds, binding was measured with $100 \mathrm{nM}$ mAbs for 2 minutes, and dissociation was measured in PBS for 2 minutes.

Antibody Scale Up and Purification. Hybridomas were adapted to DMEM (Corning Inc, Corning, NY) supplemented with $10 \%$ fetal bovine serum, hypoxanthine/thymidine (Sigma), and 2-mercaptoethanol and inoculated into Integra Celline 1000 bioreactors (Wheaton, Millville, $\mathrm{NJ})$. Supernatant containing secreted mAbs was purified by affinity chromatography with recombinant Protein A Sepharose (GE Healthcare, Chicago, IL). Antibody was sterilized by $0.2 \mu \mathrm{m}$ filtration, aliquoted in preservative-free PBS, $\mathrm{pH} 7.2$, and stored at $4^{\circ} \mathrm{C}$. Purified mAbs were analyzed by SDS-PAGE and dynamic light scattering (see Supplemental Material for additional methods).

Drug Challenges and Pharmacokinetics. Mice were passively immunized with control antibodies (Gammagard, Baxalta Inc) or antiopioid ( $\alpha$-opioid) mAbs (e.g., $\alpha$-oxycodone mAb) in sterile PBS, $40-80 \mathrm{mg} / \mathrm{kg}$ as indicated in figure legends. To determine bioavailability and serum stability of mAbs, approximately $50 \mu \mathrm{l}$ of blood was collected by facial vein sampling at least 1 hour prior to drug challenge. Mice were injected subcutaneously with $5.0 \mathrm{mg} / \mathrm{kg}$ oxycodone, $1.0 \mathrm{mg} / \mathrm{kg}$ heroin, or 0.1 or $0.5 \mathrm{mg} / \mathrm{kg}$ fentanyl, and antinociception was evaluated by latency to respond on a hot plate set to $54^{\circ} \mathrm{C}$ (Columbus Instruments, Columbus, $\mathrm{OH}$ ) at 30 minutes postinjection. Antinociception was reported as percent maximum possible effect and was calculated as (latency postinjection - baseline latency)/ $(60-$ baseline latency $) \times 100$. At 31 minutes postinjection, mice were euthanized by $\mathrm{CO}_{2}$ inhalation and decapitated, and brain and blood were collected for drug concentration analysis. Oxycodone concentrations were measured by gas chromatography-mass spectrometry (GC-MS) as described by Pravetoni et al. (2013), and concentrations of heroin, 6-acetyl morphine (6-AM), and morphine were analyzed by LCMS as previously described (Raleigh et al., 2013). Fentanyl concentrations were measured using a triple quadrupole Agilent G6470A LCMS/MS system (Santa Clara, CA), and data acquisition and peak integration were performed using MassHunter software (Tokyo, Japan). In fentanyl challenge studies, mice were also evaluated for heart rate, oxygen saturation, and breath rate using a MouseOx Plus pulse oximeter (Starr Life Sciences, Oakmont, PA). Rats were passively immunized with $60 \mathrm{mg} / \mathrm{kg} \alpha$-fentanyl mAbs i.p. and 24 hours later challenged with $0.1 \mathrm{mg} / \mathrm{kg}$ fentanyl s.c.. Antinociception, oxygen saturation and heart ratewere measured every 15 minutes postinjection

Statistical Analysis. Statistical analysis was performed using Prism (GraphPad, La Jolla, CA). Mean percent maximum possible effect, drug concentrations, oxygen saturation, heart rate (beats per minute), and breath rate (breaths per minute) were analyzed by ordinary one-way ANOVA followed by post hoc analysis by Tukey's multiple comparisons test, or by two-way ANOVA followed by Sidak's multiple comparisons test when appropriate for measurements over time.

\section{Results}

Antigen-Based Magnetic Enrichment Provides a High-Throughput Platform for Generation of Hybridomas Expressing Antiopioid mAbs. Murine hybridomas expressing $\alpha$-opioid mAbs were generated after active immunization with the cognate lead $\alpha$-opioid conjugate vaccines (see Supplemental Table 1). To isolate $\alpha$-oxycodone mAbs, a vaccine consisting of an oxycodone-based hapten (OXY, Fig. 1B) conjugated to the sKLH carrier protein (OXY-sKLH) was chosen for immunization. The OXY-sKLH vaccine has previously been shown to elicit protective titers in rats (Raleigh et al., 2018) and OXY-specific B cells in mice (Laudenbach et al., 2015). A magnetic enrichment strategy was used to isolate opioid-specific B cells prior to hybridoma fusion (see scheme in Fig. 1A). To minimize coisolation of carrier-specific splenocytes, OXY was conjugated to PE and used with anti-PE magnetic microbeads for enrichment of OXY-specific cells. Enrichment reduced the number of cells to be fused from approximately $3 \times 10^{8}$ total splenocytes to $3.8 \times 10^{7}$ enriched cells, and fusion of the OXY-specific enriched cell pool with $\mathrm{Sp} 2 / 0$ cells resulted in isolation of approximately $30 \mathrm{OXY}$ positive clones from a screening of 294 colonies $(>10 \%)$.

Because previous research indicates that depletion of interleukin-4 (IL-4) with neutralizing antibodies enhances vaccine efficacy (Laudenbach et al., 2018), a second cohort of mice was immunized with OXY-sKLH in conjunction with a neutralizing rat anti-murine IL-4 mAb ( $\alpha$-IL-4, clone 11B11, BioXCell, Lebanon, $\mathrm{NH}$ ). An additional 12 hybridomas expressing $\alpha$-oxycodone mAbs were isolated from this fusion and were evaluated by ELISA to determine the IgG subclasses of expressed mAbs (Supplemental Fig. 1). Whereas all clones isolated from immunization with aluminum adjuvant alone expressed $\operatorname{IgG}_{1} \alpha$-oxycodone mAbs, immunization with aluminum in combination with $\alpha$-IL-4 allowed isolation of several clones expressing $\alpha$-oxycodone $\operatorname{IgG}_{2 a}$ mAbs, suggesting that choice of adjuvant impacts the profile of mAbs generated from hybridomas. In addition, these data further support use of molecular adjuvants or immunomodulators such as the $\alpha$-IL-4 mAbs to improve the quality of the polyclonal antibody responses and the likelihood of isolating mAbs of a desired IgG subclass.

For generation of $\alpha$-heroin and $\alpha$-fentanyl mAbs, mice were immunized with conjugate vaccines containing either a morphine-based hapten (Fig. 1C) or a fentanyl-based hapten (Fig. 1D) conjugated to sKLH. Active immunization with M-sKLH has been previously shown to generate polyclonal antibody response targeting heroin and its active metabolites 6-AM and morphine in mice and rats (Raleigh et al., 2013). Active immunization with $\mathrm{F}$-sKLH has been shown to protect against fentanyl-induced respiratory depression and bradycardia in rats (Raleigh et al., 2019). A similar magnetic enrichment strategy as that for $\alpha$-oxycodone mAbs was used to isolate hybridomas expressing $\alpha$-heroin and $\alpha$-fentanyl mAbs, resulting in a reduction in splenocytes available for fusion to approximately $2 \times 10^{7}$ and $2.5 \times 10^{7}$ enriched cells, respectively, and yielding approximately $5 \%$ of hybridoma clones producing mAbs specific for the immunizing antigen.

Initial Biophysical Characterization and Scalability. Hybridomas expressing $\alpha$-opioid mAbs were initially cultured in $10 \mathrm{~cm}$ dishes using ClonaCell-HY medium, and mAbs were purified from supernatant at a $10 \mathrm{mg}$ scale for initial in vitro characterization. Selected hybridoma clones were then transferred across laboratory sites and adapted to growth in Integra CL 1000 bioreactors, which yielded up to $250 \mathrm{mg}$. Lead mAbs were purified and characterized by dynamic light scattering and SDS-PAGE to evaluate aggregation state and molecular weight (Supplemental Fig. 2). These data support scalability of the lead mAbs generated in this study, and preliminary feasibility of technology transfer to a contract research organization with the goal of scaling up the mAb production 
A

A X-PE + anti-PE magnetic bead

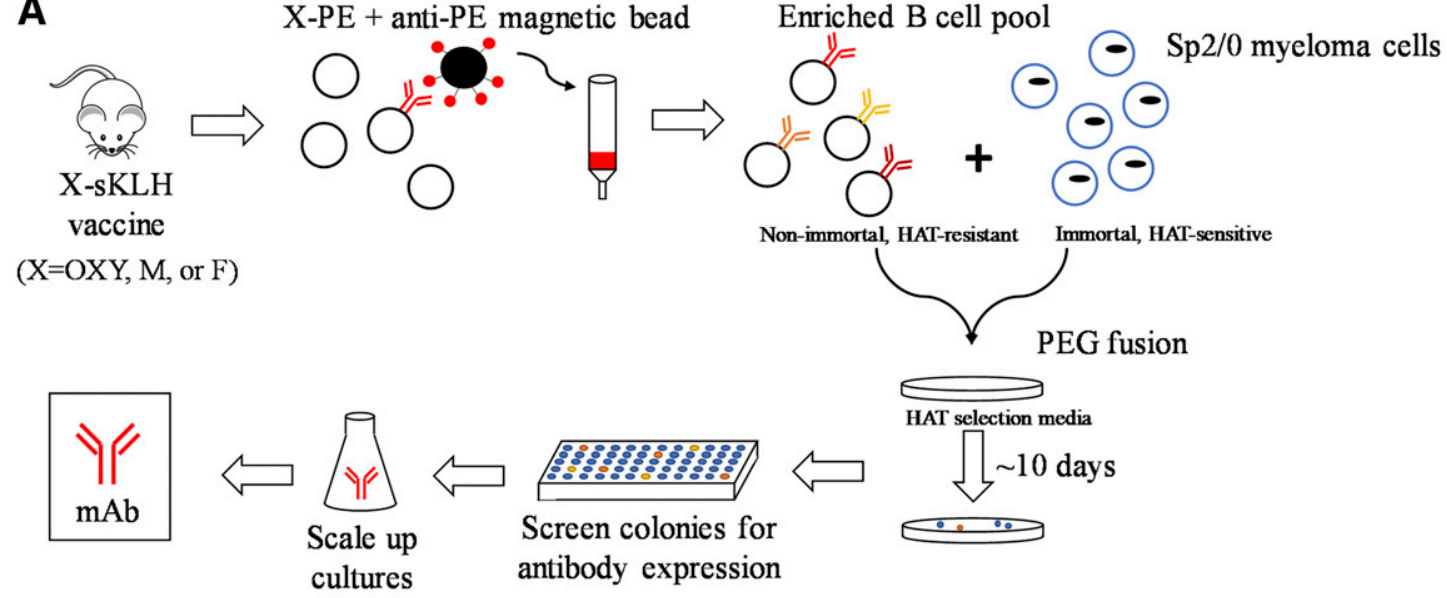

B

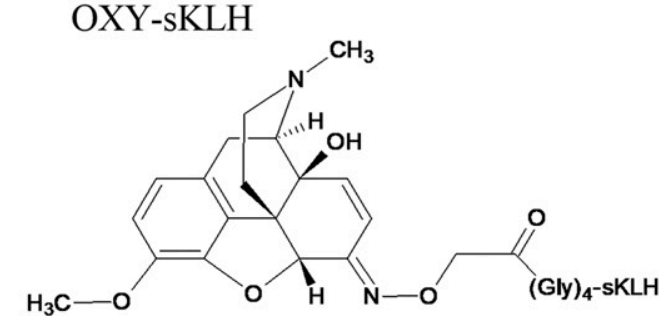

C

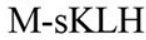

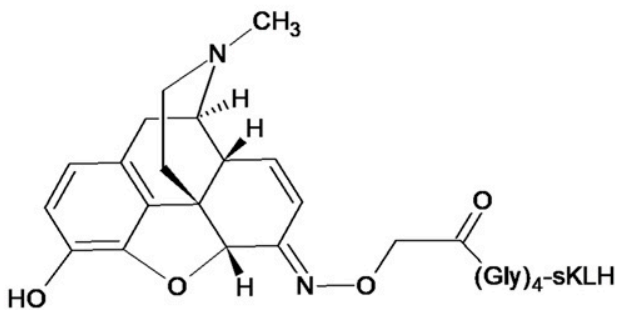

D F-sKLH<smiles>CCC(=O)C(CCCC(=O)NCCN1CCC(N(C(=O)CC)c2ccccc2)CC1)C(=O)O</smiles>

Fig. 1. B cell-based platform for generating $\alpha$-opioid hybridomas via magnetic enrichment. (A) Workflow for hybridoma generation. Antigen-specific cells from immunized mice are selected by magnetic enrichment for polyethylene glycol (PEG) fusion with Sp2/0 myeloma cells; hypoxanthineaminopterin-thymidine (HAT)-resistant colonies are transferred to plates and screened for expression of antiopioid antibodies by ELISA. Structures of haptens used for immunization for $\alpha$-oxycodone (B), $\alpha$-heroin (C), and $\alpha$-fentanyl (D) hybridomas.

to support late-stage characterization and in vivo studies in large animal models.

In Vivo Efficacy of $\alpha$-Oxycodone mAbs. Selected $\alpha$-oxycodone mAbs were purified from the hybridoma supernatant, and their relative affinity for oxycodone was determined by competitive ELISA (Fig. 2A). Antioxycodone mAbs exhibited $\mathrm{IC}_{50}$ within the $10 \mathrm{nM}$ to $1 \mu \mathrm{M}$ range. Several clones were selected for further scale up and characterization; whereas clone HY1-3E3 exhibited the highest in vitro affinity, the isolated mAbs exhibited poor in vivo efficacy in initial tests (Supplemental Fig. 3). Therefore, two clones with robust mAb expression were selected as leads, including one $\operatorname{IgG}_{1}$ clone (HY1-3G8) and one IgG $_{2 \mathrm{a}}$ clone (HY2-A12).

To evaluate whether an $\alpha$-oxycodone mAb would reduce the effects of oxycodone in vivo, mice were passively immunized with purified mAbs 24 hours before a $5.0 \mathrm{mg} / \mathrm{kg}$ oxycodone challenge. Doses of either 40 or $80 \mathrm{mg} / \mathrm{kg}$ HY1-3G8 significantly reduced antinociception in mice compared with control Abs (Fig. 2B) at thirty minutes after administration of the drug. Treatment with $40 \mathrm{mg} / \mathrm{kg}$ HY2-A12 showed a trend toward reduced antinociception $(P=0.065)$. At completion of the behavioral assessment, mice were euthanized, and the concentration of oxycodone in the brain and serum was measured by GC-MS. Passive immunization with $\alpha$-oxycodone mAbs increased retention of oxycodone in serum compared to control (Fig. 2C), and the effect was more pronounced in mice receiving $80 \mathrm{mg} / \mathrm{kg}$ of mAbs. Treatment with $40 \mathrm{mg} / \mathrm{kg}$ of either $\mathrm{IgG}_{1}$ or $\mathrm{IgG}_{2 \mathrm{a}}$ $\alpha$-oxycodone mAbs reduced brain distribution of drug by approximately $49 \%$ (Fig. 2D), whereas $80 \mathrm{mg} / \mathrm{kg}$ of $\mathrm{IgG}_{1} \alpha$-oxycodone mAbs reduced brain distribution by $65 \%$. These data suggest that IgG subclass may be not be a major contributor to antibody efficacy in vivo and that mAb efficacy is dose dependent. 
A

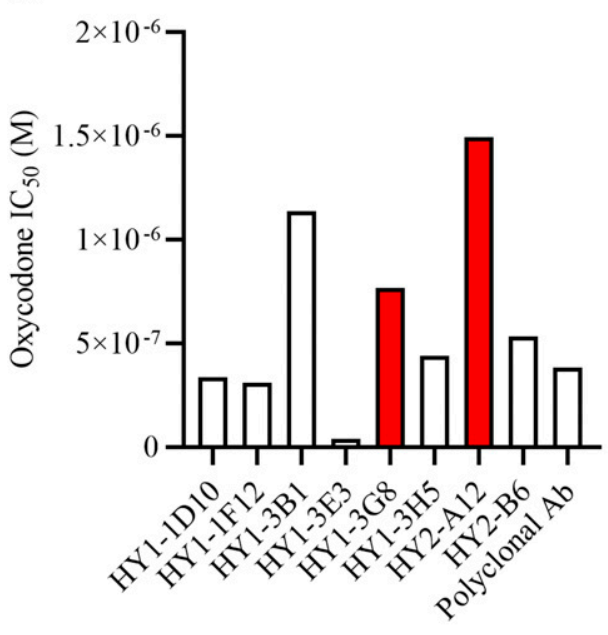

C

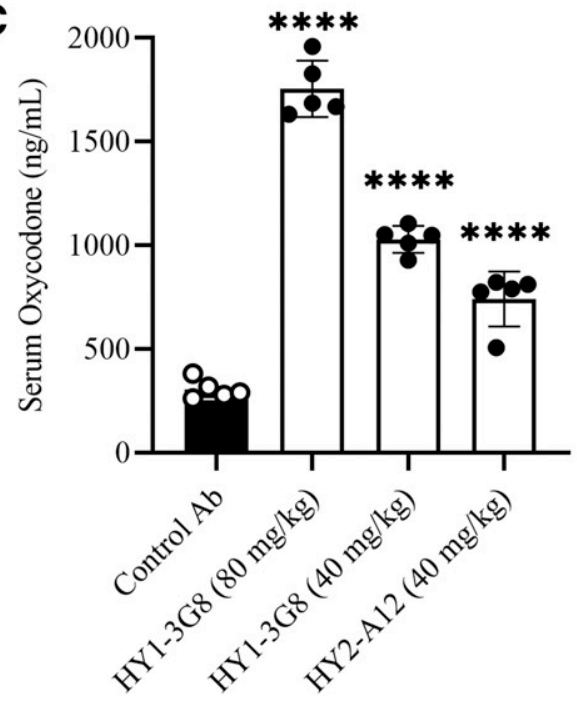

B
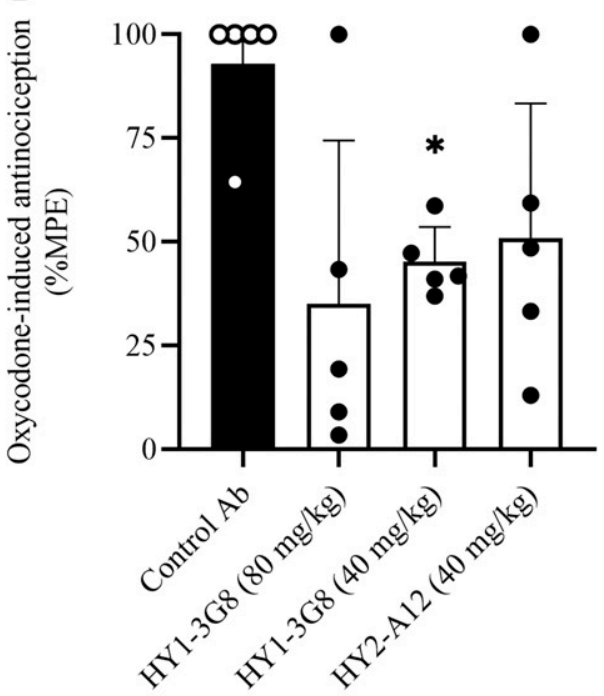

D

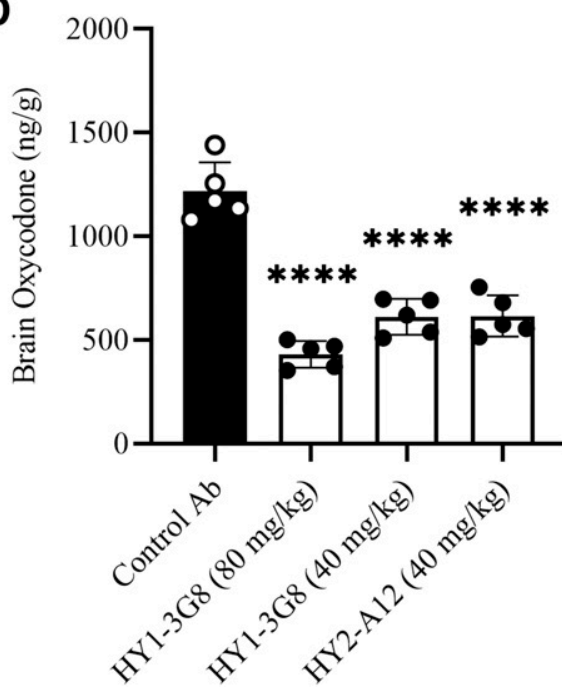

Fig. 2. Characterization and efficacy of $\alpha$-oxycodone mAbs. (A) Affinity for oxycodone of $\alpha$-oxycodone mAbs was determined by competitive ELISA. (B-D) Passive immunization with $\alpha$-oxycodone mAbs reduces oxycodone-induced antinociception and oxycodone distribution to the brain. Mice ( $n=5$ /group) were passively immunized with 40 or $80 \mathrm{mg} / \mathrm{kg}$ $\alpha$-oxycodone mAbs i.p., and challenged with $5.0 \mathrm{mg} / \mathrm{kg}$ oxycodone s.c.. (B) Antinociception was evaluated by latency to respond on a hot plate at 30 minutes postdrug challenge, and oxycodone levels in serum $(\mathrm{C})$ and brain $(\mathrm{D})$ were determined by GC-MS. \%MPE, percent maximum possible effect. Mean \pm S.D.; ${ }^{*} P<0.05$; $* * * * P<0.0001$
In Vivo Efficacy of $\boldsymbol{\alpha}$-Heroin mAbs. For $\alpha$-heroin mAbs, relative affinity of purified mAbs was evaluated by competitive ELISA using plates coated with M-BSA.Free morphine was used as competitor for screening because heroin is relatively unstable and quickly degrades to 6-AM and morphine. Three clones exhibited $\mathrm{IC}_{50}<2 \mathrm{nM}$ (Fig. 3A), and HY4$1 F 9$ was chosen for scale up and in vivo characterization. Mice were passively immunized with purified $\alpha$-heroin mAbs and 24 hours later challenged with $1 \mathrm{mg} / \mathrm{kg}$ heroin. Treatment with mAbs reduced heroin-induced antinociception (Fig. 3B), but the effect was not statistically significant $(P=0.086$ for $40 \mathrm{mg} / \mathrm{kg} ; P=0.127$ for $80 \mathrm{mg} / \mathrm{kg}$ ). Because heroin is rapidly metabolized in vivo to active metabolites 6-AM and morphine, the concentrations of these metabolites in the brain and serum 30 minutes postchallenge were measured by LC-MS as a correlate of drug distribution. Measured levels of heroin were near or below the lower limit of quantitation and excluded from analysis. At a dose of $1 \mathrm{mg} / \mathrm{kg}$ heroin, pretreatment with $40 \mathrm{mg} / \mathrm{kg}$ of $\alpha$-heroin mAbs reduced brain distribution of heroin metabolites by $35 \%$, and $80 \mathrm{mg} / \mathrm{kg} \mathrm{mAbs}$ reduced distribution by $57 \%$ of control (Fig. 3D).
To investigate the distribution of $\alpha$-opioid mAbs after passive immunization, $40 \mathrm{mg} / \mathrm{kg}$ of the lead $\alpha$-heroin $\mathrm{mAb}$ HY4-1F9 was administered to mice either subcutaneously or intraperitoneally, and blood was sampled at intervals after administration to determine concentration of HY4-1F9 in serum (Supplemental Fig. 4B). Importantly, the resulting serum mAb concentrations were equivalent between these routes, suggesting that both subcutaneous and intraperitoneal injection are viable for delivery of mAbs and supporting use of the more convenient subcutaneous delivery for $\alpha$-opioid prophylaxis in potential clinical applications.

In Vivo Efficacy of $\alpha$-Fentanyl mAbs. Relative affinities of $\alpha$-fentanyl mAbs were measured by BLI, with the highest affinity mAb HY6-F9 exhibiting a dissociation constant of $\sim 0.5 \mathrm{nM}$ (Fig. 4A). The two lead mAbs selected for scale up included HY6-B5 and HY6-F9, which were $\mathrm{IgG}_{1}$ and $\mathrm{IgG}_{2 \mathrm{a}}$ subtypes, respectively (Supplemental Fig. 1). To evaluate the efficacy of these mAbs, male and female mice were passively immunized with $40 \mathrm{mg} / \mathrm{kg}$ of either HY6-B5 or HY6F9. Because fentanyl-induced respiratory depression is a major contributor to overdose fatalities (Fox et al., 2018), the 
A

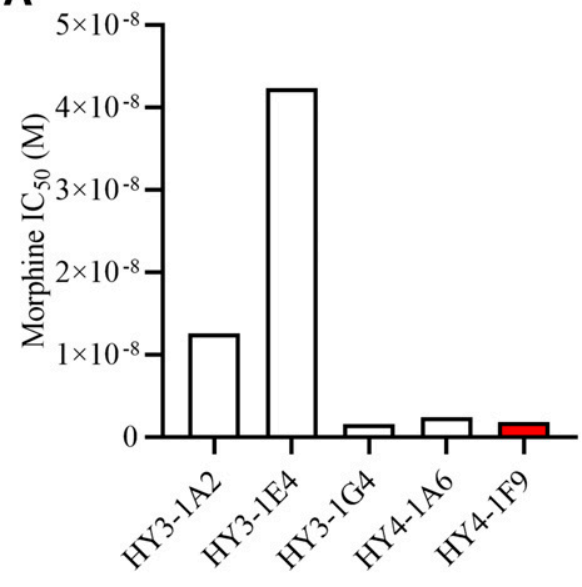

C

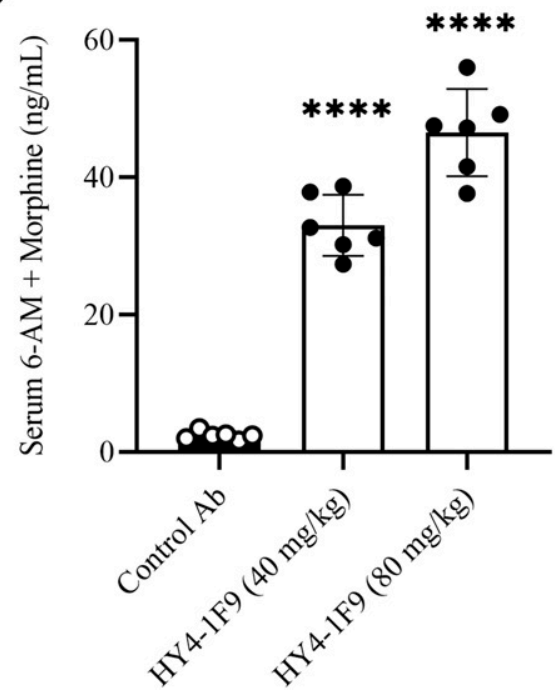

B
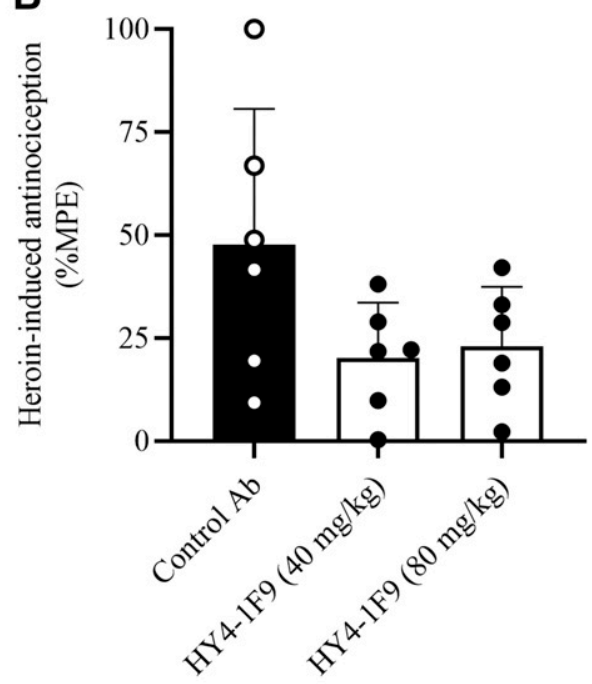

D

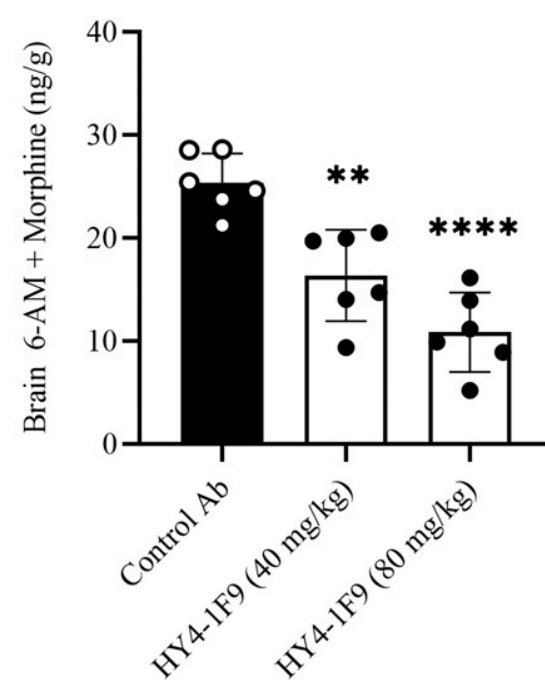

Fig. 3. Characterization and efficacy of $\alpha$-heroin mAbs. (A) Relative affinities of $\alpha$-heroin mAbs were determined by competitive binding ELISA using morphine as a competitor. (B-D) Passive immunization with $\alpha$-heroin mAbs reduces heroin distribution to the brain. Mice $(n=6 /$ group) were passively immunized with 40 or $80 \mathrm{mg} / \mathrm{kg} \alpha$-heroin mAbs i.p. and challenged with $1.0 \mathrm{mg} / \mathrm{kg}$ heroin s.c. (B) Antinociception was evaluated by latency to respond on a hot plate 30 minutes postdrug injection.Shown heroin metabolites 6-AM and morphine in serum (C) and brain (D) were determined by LC-MS. \% MPE, percent maximum possible effect. Mean \pm S.D.; $* * P<0.01 ; * * * P<0.0001$. effects of fentanyl on respiratory behavior were measured 30 minutes after administration of $0.1 \mathrm{mg} / \mathrm{kg}$ fentanyl. Passive immunization with either HY6-B5 or HY6-F9 reduced fentanyl antinociception (Fig. 4B), and HY6-F9 prevented fentanyl-induced suppression of breath and heart rate (Fig. 4, C and D) compared with predrug baseline values. Female mice treated with control Abs showed slightly greater fentanyl-induced antinociception than male mice treated with control Abs, but no other statistically significant differences between male and female mice in the same treatment group were observed, and HY6-F9 significantly reduced fentanylinduced antinociception and bradycardia in both male and female mice (Supplemental Fig. 5).

To evaluate the effect of passive immunization at higher doses of fentanyl, separate cohorts of male and female mice were passively immunized with $40 \mathrm{mg} / \mathrm{kg}$ HY6-F9 and challenged with $0.5 \mathrm{mg} / \mathrm{kg}$ fentanyl. At this dose of fentanyl, treatment with HY6-F9 increased oxygen saturation and reduced brain concentration of fentanyl by $85 \%$ compared to control (Fig. 4, E and F).

Because HY6-F9 was effective in reducing the effects of fentanyl in mice, in vivo efficacy of this mAb was also evaluated in rats. Rats were passively immunized with $60 \mathrm{mg} / \mathrm{kg} \mathrm{mAbs}$ and challenged with $0.1 \mathrm{mg} / \mathrm{kg}$ fentanyl, and antinociception and respiratory behavior were measured every 15 minutes for 1 hour. Pretreatment with the $\alpha$-fentanyl mAb was effective at reducing antinociception (Fig. 5A) and preventing loss of oxygen saturation and heart rate (Fig. 5, B and C) after administration of fentanyl.

\section{Discussion}

Monoclonal antibodies and vaccines against drugs of abuse or other chemical threats offer a unique tool with potential applications for treatment of OUD and prevention of overdose or toxicity. Here, we describe the development of mAbs against three opioid targets (oxycodone, heroin, and fentanyl), and demonstrate their efficacy in vivo. The magnetic enrichment strategy used to develop the mAbs generated in this study reduced the number of total hybridoma clones 10 -fold, simplifying the screening process. Similar methods have been successfully applied to generation of hybridomas against protein and peptide targets, including major histocompatibility complex-peptide complexes (Spanier et al., 2016). However, 
A

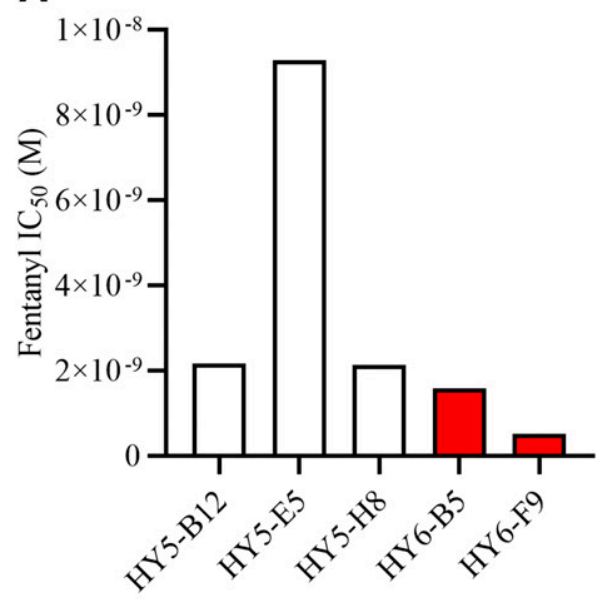

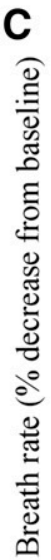

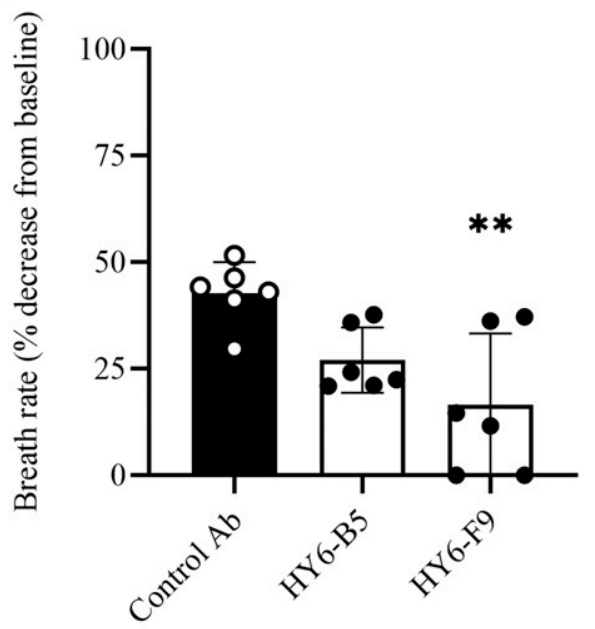

E

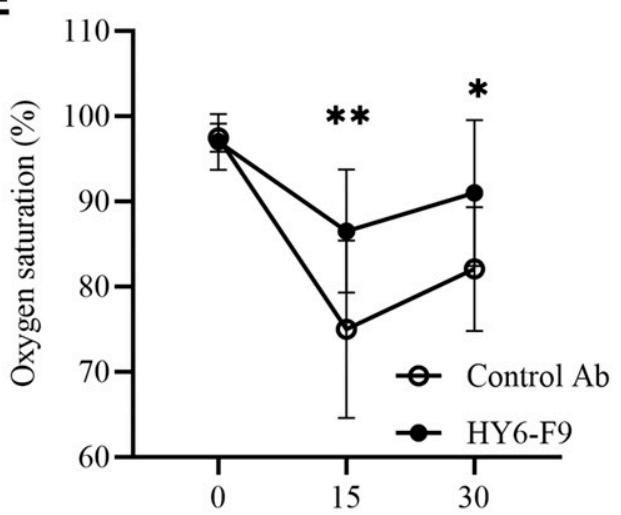

B

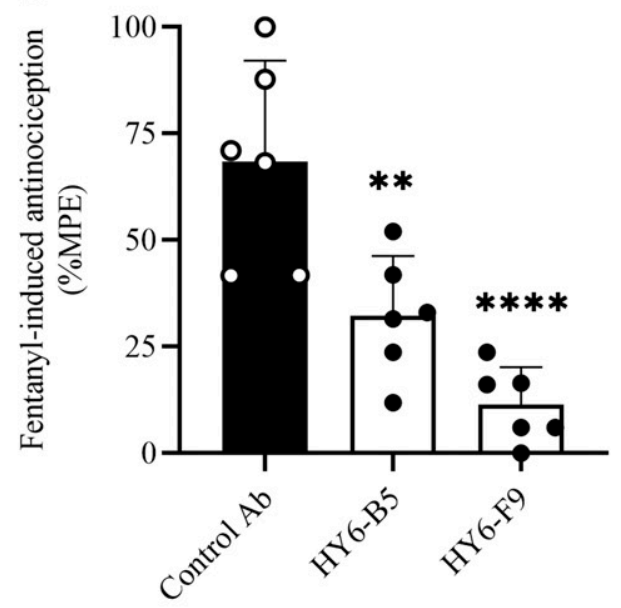

D

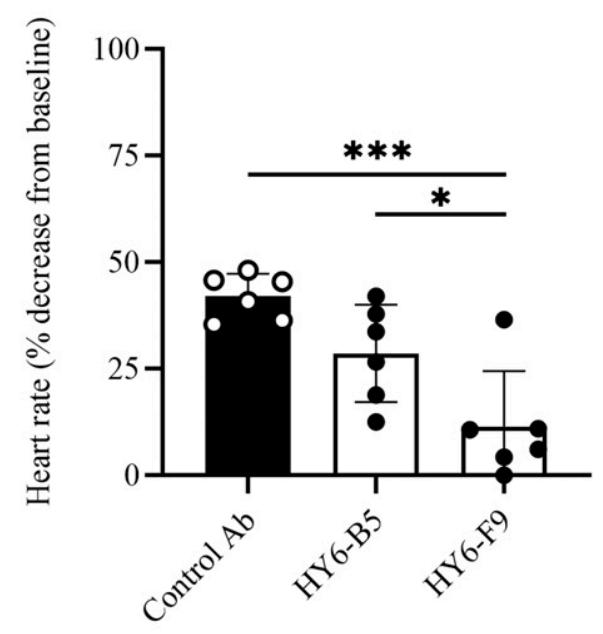

$\mathbf{F}$

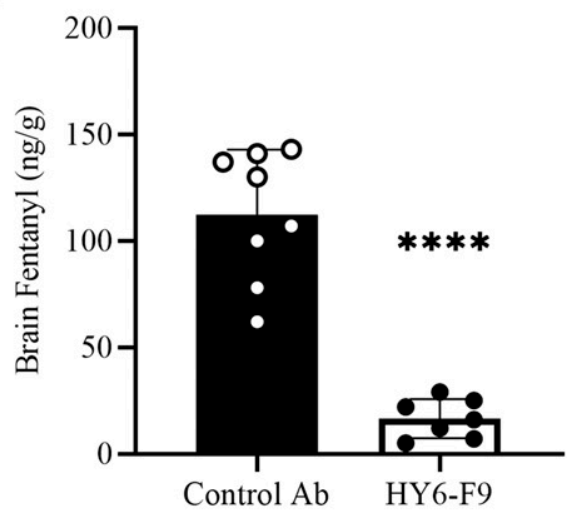

Fig. 4. Characterization and efficacy of $\alpha$-fentanyl mAbs. (A) Dissociation constants of $\alpha$-fentanyl mAbs were determined by biolayer interferometry. (B-D) Passive immunization with $\alpha$-fentanyl mAbs reduces fentanyl-induced antinociception, respiratory depression, and bradycardia. Mice ( $n=3$ male and 3 female per group) were passively immunized with $40 \mathrm{mg} / \mathrm{kg} \alpha$-fentanyl mAbs i.p. and challenged with $0.1 \mathrm{mg} / \mathrm{kg}$ fentanyl s.c.. (B) Antinociception was evaluated by latency to respond on a hot plate. Breath rate $(C)$ and heart rate (D) were measured by pulse oximetry at 30 minutes postdrug injection. (E - F) In a separate experiment, mice $(n=4$ male and 4 female per group) were passively immunized with $40 \mathrm{mg} / \mathrm{kg} \alpha$-fentanyl mAbs and challenged with $0.5 \mathrm{mg} / \mathrm{kg}$ fentanyl. (E) Oxygen saturation, and (F) brain fentanyl concentrations measured by LC-MS at 30 minutes postdrug injection. \%MPE, percent maximum possible effect. Mean \pm S.D.; $* * P<0.01 ; * * * P<0.001 ; * * * * P<0.0001$

Time (min)

this is the first report to our knowledge to apply magnetic enrichment of splenocytes prior to hybridoma fusion to the generation of $\mathrm{mAbs}$ against a chemical target.

Selected mAbs isolated using this approach exhibited both in vitro binding capability to their target drug and in vivo efficacy in reducing behavioral and pharmacologic effects of opioids. In comparison with active vaccination, which may require weeks to months with multiple boosts to mount a protective immune response, passive immunization with $\alpha$-opioid mAbs offers the benefit of greater control over the dose, affinity, and peak timing of protective Abs. However, because efficacy of mAbs and vaccines against opioids depends 


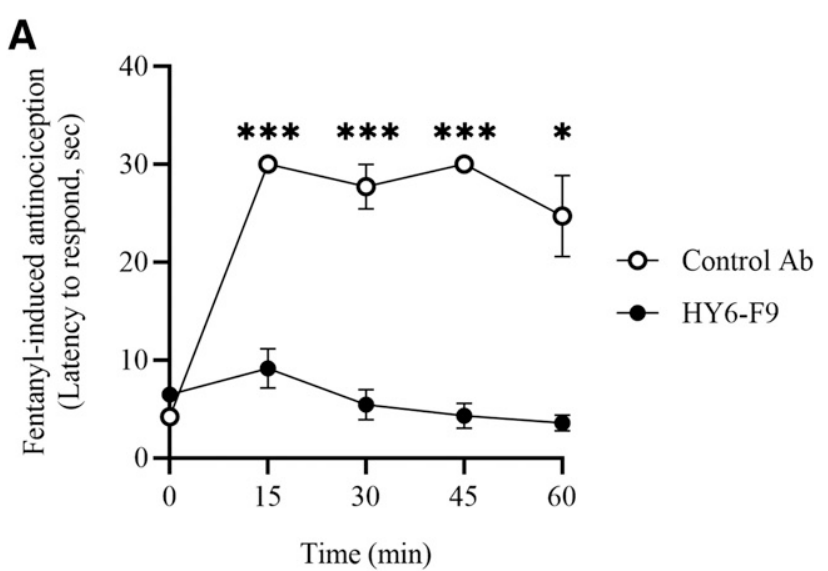

B
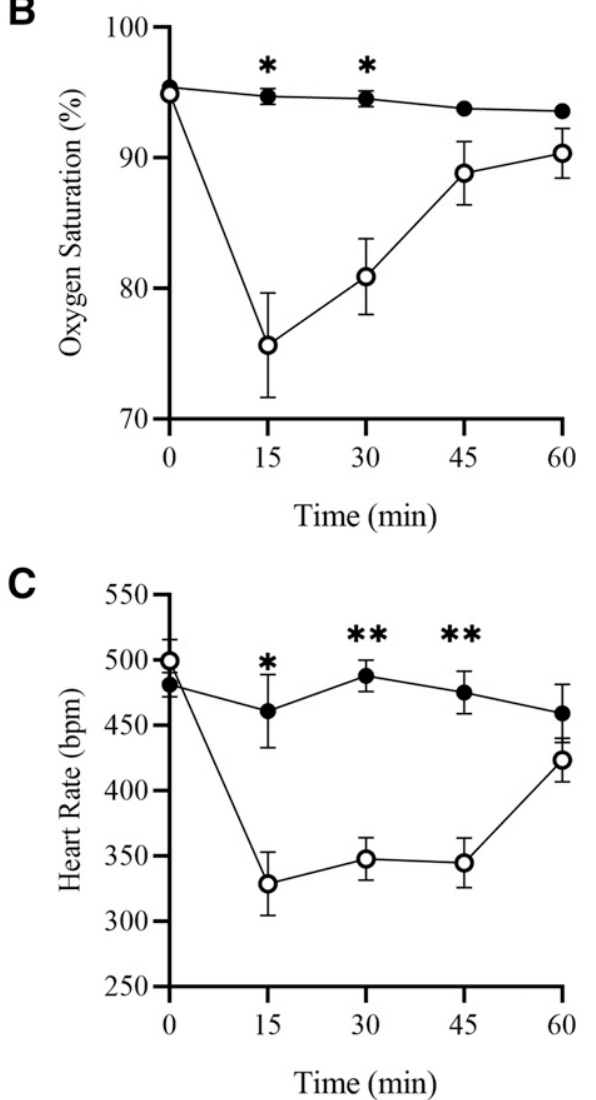

Fig. 5. Efficacy of $\alpha$-fentanyl mAbs in rats. Rats ( $n=4$ /group) were passively immunized with $60 \mathrm{mg} / \mathrm{kg} \alpha$-fentanyl mAbs i.p. and challenged with $0.1 \mathrm{mg} / \mathrm{kg}$ fentanyl s.c. Antinociception by latency to respond on a hot plate (A), oxygen saturation (B), and heart rate (C) were evaluated every 15 minutes up to 1 hour postdrug injection. Mean \pm S.D.; $* P<0.05 ; * * P<$ $0.01 ; * * * P<0.001$.

on binding and sequestration of the drug of interest in serum, favorable stoichiometric ratios of $\mathrm{Ab}$ binding sites to drug in serum are required to achieve the reduction in brain distribution necessary to block pharmacological and physiological effects. Typically, doses of $30-120 \mathrm{mg} / \mathrm{kg}$ of an $\alpha$-opioid mAb are required for passive immunization (Smith et al., 2019), and efficacy is dose-dependent with larger mAb doses offering greater protection against opioid intoxication (Kvello et al., 2016,2019 ). The doses of $40-80 \mathrm{mg} / \mathrm{kg}$ shown here result in $\mathrm{mAb}$ serum concentrations comparable to levels of polyclonal Abs achieved with their corresponding vaccinating antigens
OXY-sKLH and M-sKLH (Supplemental Fig. 3) and similar in vivo efficacy (Raleigh et al., 2014, 2017). Although the relatively high manufacturing cost of $\mathrm{mAb}$ production can be a significant limitation to clinical translation of mAb-based therapies for OUD due to high dosing requirements, advances in manufacturing technologies for biologics (Buyel et al., 2017; Diamos et al., 2020) are expected to facilitate cost-effective $\mathrm{mAb}$ production in the future.

For both the $\alpha$-oxycodone mAbs (Fig. 2D) and the $\alpha$-heroin mAbs (Fig. 3D), a dose of $80 \mathrm{mg} / \mathrm{kg}$ produced a greater reduction in brain distribution in comparison with a lower dose of $\mathrm{mAb}$ and a corresponding increase in the concentration of drug sequestered in the serum compartment (Figs. 2C and 3C). Notably, $\alpha$-heroin mAbs appeared less effective than $\alpha$-oxycodone mAbs in terms of reducing brain concentration of drug, despite a 5-fold lower drug dose and a higher apparent affinity for the $\alpha$-heroin mAbs. The finding that Abs against oxycodone are more effective than those against heroin is consistent with trends observed after active immunization with OXY-sKLH and M-sKLH, and efficacy depends on the route of oxycodone or heroin administration (Raleigh et al., 2018). Because mAbs can theoretically only interact with circulating drugs in the serum compartment, whereas circulating unbound drug is subject to metabolism and tissue distribution effects, it can be difficult to predict mAb doses required for efficacy against various opioids. For example, a dose of $5 \mathrm{mg} / \mathrm{kg}$ oxycodone is in 30 -fold excess of available binding sites when considering the total dose ratio, whereas measured serum concentrations of oxycodone and mAbs estimate that the amount of drug in the serum compartment is $\sim 50 \%-60 \%$ that of available binding sites. Regardless, $40 \mathrm{mg} / \mathrm{kg} \mathrm{mAbs}$ were sufficient to significantly impact physiologic responses to oxycodone (Fig. 2B) and reduce brain concentration $\sim 50 \%$, whereas brain concentration of heroin after $1 \mathrm{mg} / \mathrm{kg}$ challenge (corresponding to a 5-fold heroin:mAb binding sites total dose ratio) was reduced $\sim 35 \%$ (Fig. 3D).

Monoclonal antibodies against fentanyl and fentanyl analogs are of particular interest to public health due to their high potency. Because fentanyl-induced respiratory depression and bradycardia are implicated in overdose fatalities, heart rate and respiration were used as primary measures of $\alpha$-fentanyl $\mathrm{mAb}$ efficacy. Passive vaccination was effective at reducing toxic effects of fentanyl in both mice and rats (Figs. 4 and 5), supporting the potential of mAbs as a therapeutic to protect against fentanyl toxicity. However, at the higher fentanyl dose $(0.5 \mathrm{mg} / \mathrm{kg})$ in mice, mAbs reduced respiratory depression compared with control Abs but were less protective against fentanyl-induced antinociception and bradycardia despite an $85 \%$ reduction in brain distribution in mAb-treated mice. Although further studies with larger doses of $\alpha$-fentanyl mAbs will be required to establish whether mAbs can protect or rescue from high plasma concentrations of fentanyl that may be encountered in an overdose scenario, other studies have successfully demonstrated mAb-based protection from potentially lethal fentanyl doses (Smith et al., 2019). In this context, mAbs could act as either a standalone treatment or as a supplement to opioid antagonists (e.g., naloxone) for overdose treatment and prevention. Preclinical efficacy of these opioid-targeting biologics has been demonstrated in multiple models and with various dose ranges and routes of administration, supporting further clinical development of this therapeutic approach. 


\section{Acknowledgments}

The authors thank Jennifer Vigliaturo and Michael Raleigh for technical support and discussions.

\section{Authorship Contributions}

Participated in research design: Baehr, AuCoin, Pravetoni.

Conducted experiments: Baehr, Huseby Kelcher, Khaimraj, Reed,

Pandit.

Contributed new reagents or analytic tools: Averick.

Wrote or contributed to the writing of the manuscript: Baehr, Pravetoni.

\section{References}

Baruffaldi F, Kelcher AH, Laudenbach M, Gradinati V, Limkar A, Roslawski M, Birnbaum A, Lees A, Hassler C, Runyon S, et al. (2018) Preclinical efficacy and characterization of candidate vaccines for treatment of opioid use disorders using clinically viable carrier proteins. Mol Pharm 15:4947-4962.

Boonyaratanakornkit J and Taylor JJ (2019) Techniques to study antigen-specific B cell responses. Front Immunol 10:1694.

Bremer PT and Janda KD (2017) Conjugate vaccine immunotherapy for substance use disorder. Pharmacol Rev 69:298-315.

Bremer PT, Schlosburg JE, Banks ML, Steele FF, Zhou B, Poklis JL, and Janda KD (2017) Development of a clinically viable heroin vaccine. J Am Chem Soc 139: 8601-8611.

Buyel JF, Twyman RM, and Fischer R (2017) Very-large-scale production of antibodies in plants: the biologization of manufacturing. Biotechnol Adv 35:458-465.

Centers for Disease Control and Prevention (May 5, 2020) Opioid overdose. https:// www.cdc.gov/drugoverdose/data/index.html

Cornuz J, Zwahlen S, Jungi WF, Osterwalder J, Klingler K, van Melle G, Bangala Y, Guessous I, Müller P, Willers J, et al. (2008) A vaccine against nicotine for smoking cessation: a randomized controlled trial. PLoS One 3:e2547.

Diamos AG, Hunter JGL, Pardhe MD, Rosenthal SH, Sun H, Foster BC, DiPalma MP, Chen Q, and Mason HS (2020) High level production of monoclonal antibodies using an optimized plant expression system. Front Bioeng Biotechnol 7:472.

Fox BS, Kantak KM, Edwards MA, Black KM, Bollinger BK, Botka AJ, French TL, Thompson TL, Schad VC, Greenstein JL, et al. (1996) Efficacy of a therapeutic cocaine vaccine in rodent models. Nat Med 2:1129-1132.

Fox LM, Hoffman RS, Vlahov D, and Manini AF (2018) Risk factors for severe respiratory depression from prescription opioid overdose. Addiction 113: 59-66.

Han Y, Yan W, Zheng Y, Khan MZ, Yuan K, and Lu L (2019) The rising crisis of illicit fentanyl use, overdose, and potential therapeutic strategies. Transl Psychiatry 9: 282.

Ho IY, Bunker JJ, Erickson SA, Neu KE, Huang M, Cortese M, Pulendran B, and Wilson PC (2016) Refined protocol for generating monoclonal antibodies from single human and murine B cells. J Immunol Methods 438:67-70.

Jarvis BP, Holtyn AF, Subramaniam S, Tompkins DA, Oga EA, Bigelow GE, and Silverman K (2018) Extended-release injectable naltrexone for opioid use disorder: a systematic review. Addiction 113:1188-1209.

Kashanian S, Shams A, Ghahremani H, and Paknejad M (2015) Preparation and characterization of a monoclonal antibody against morphine. Monoclon Antib Immunodiagn Immunother 34:270-274.

Keyler DE, Roiko SA, Benlhabib E, LeSage MG, St Peter JV, Stewart S, Fuller S, Le CT, and Pentel PR (2005) Monoclonal nicotine-specific antibodies reduce nicotine distribution to brain in rats: dose- and affinity-response relationships. Drug Metab Dispos 33:1056-1061.

Kosten TR, Domingo CB, Shorter D, Orson F, Green C, Somoza E, Sekerka R, Levin FR, Mariani JJ, Stitzer M, et al. (2014) Vaccine for cocaine dependence: a randomized double-blind placebo-controlled efficacy trial. Drug Alcohol Depend 140: 42-47.

Kvello AMS, Andersen JM, Øiestad EL, Mørland J, and Bogen IL (2016) Pharmacological effects of a monoclonal antibody against 6-monoacetylmorphine upon heroin-induced locomotor activity and pharmacokinetics in mice. J Pharmacol Exp Ther 358:181-189.

Kvello AMS, Andersen JM, Øiestad EL, Steinsland S, Aase A, Mørland J, and Bogen IL (2019) A monoclonal antibody against 6-acetylmorphine protects female mice offspring from adverse behavioral effects induced by prenatal heroin exposure. J Pharmacol Exp Ther 368:106-115.

Laudenbach M, Baruffaldi F, Robinson C, Carter P, Seelig D, Baehr C, and Pravetoni M (2018) Blocking interleukin-4 enhances efficacy of vaccines for treatment of opioid abuse and prevention of opioid overdose. Sci Rep 8:5508.

Laudenbach M, Baruffaldi F, Vervacke JS, Distefano MD, Titcombe PJ, Mueller DL, Tubo NJ, Griffith TS, and Pravetoni M (2015) The frequency of naive and earlyactivated hapten-specific B cell subsets dictates the efficacy of a therapeutic vaccine against prescription opioid abuse. J Immunol 194:5926-5936.

Lei L, Tran K, Wang Y, Steinhardt JJ, Xiao Y, Chiang C-I, Wyatt RT, and Li Y (2019) Antigen-specific single B cell sorting and monoclonal antibody cloning in Guinea pigs. Front Microbiol 10:672.

Li S, Cohen-Karni D, Kovaliov M, Tomycz N, Cheng B, Whiting D, and Averick S (2017) Synthesis and biological evaluation of fentanyl acrylic derivatives. RSC Advances 7:20015-20019.
Marckel JA, Wetzel HN, Amlal S, Amlal H, and Norman AB (2019) A recombinant humanized anticocaine monoclonal antibody alters the urinary clearance of cocaine and its metabolites in rats. Drug Metab Dispos 47:184-188.

Martell BA, Orson FM, Poling J, Mitchell E, Rossen RD, Gardner T, and Kosten TR (2009) Cocaine vaccine for the treatment of cocaine dependence in methadonemaintained patients: a randomized, double-blind, placebo-controlled efficacy trial. Arch Gen Psychiatry 66:1116-1123.

Nguyen JD, Hwang CS, Grant Y, Janda KD, and Taffe MA (2018) Prophylactic vaccination protects against the development of oxycodone self-administration. Neuropharmacology 138:292-303.

Pravetoni M (2016) Biologics to treat substance use disorders: current status and new directions. Hum Vaccin Immunother 12:3005-3019.

Pravetoni M and Comer SD (2019) Development of vaccines to treat opioid use disorders and reduce incidence of overdose. Neuropharmacology 158:107662.

Pravetoni M, Le Naour M, Harmon TM, Tucker AM, Portoghese PS, and Pentel PR (2012) An oxycodone conjugate vaccine elicits drug-specific antibodies that reduce oxycodone distribution to brain and hot-plate analgesia. J Pharmacol Exp Ther 341:225-232.

Pravetoni M, Le Naour M, Tucker AM, Harmon TM, Hawley TM, Portoghese PS, and Pentel PR (2013) Reduced antinociception of opioids in rats and mice by vaccination with immunogens containing oxycodone and hydrocodone haptens. $J \mathrm{Med}$ Chem 56:915-923.

Raleigh MD, Accetturo C, and Pravetoni M (2020) Combining a candidate vaccine for opioid use disorders with extended-release naltrexone increases protection against oxycodone-induced behavioral effects and toxicity. $J$ Pharmacol Exp Ther 374: 392-403.

Raleigh MD, Baruffaldi F, Peterson SJ, Le Naour M, Harmon TM, Vigliaturo JR, Pentel PR, and Pravetoni M (2019) A fentanyl vaccine alters fentanyl distribution and protects against fentanyl-induced effects in mice and rats. $J$ Pharmacol Exp Ther 368:282-291.

Raleigh MD, Laudenbach M, Baruffaldi F, Peterson SJ, Roslawski MJ, Birnbaum AK, Carroll FI, Runyon SP, Winston S, Pentel PR, et al. (2018) Opioid dose- and route-dependent efficacy of oxycodone and heroin vaccines in rats. $J$ Pharmacol Exp Ther 365:346-353.

Raleigh MD, Pentel PR, and LeSage MG (2014) Pharmacokinetic correlates of the effects of a heroin vaccine on heroin self-administration in rats. PLoS One 9: e115696

Raleigh MD, Peterson SJ, Laudenbach M, Baruffaldi F, Carroll FI, Comer SD, Navarro HA, Langston TL, Runyon SP, Winston S, et al. (2017) Safety and efficacy of an oxycodone vaccine: addressing some of the unique considerations posed by opioid abuse. PLoS One 12:e184876.

Raleigh MD, Pravetoni M, Harris AC, Birnbaum AK, and Pentel PR (2013) Selective effects of a morphine conjugate vaccine on heroin and metabolite distribution and heroin-induced behaviors in rats. J Pharmacol Exp Ther 344:397-406.

Robinson C, Baehr C, Schmiel SE, Accetturo C, Mueller DL, and Pravetoni M (2019) Alum adjuvant is more effective than MF59 at prompting early germinal center formation in response to peptide-protein conjugates and enhancing efficacy of a vaccine against opioid use disorders. Hum Vaccin Immunother 15:909-917.

Rzasa Lynn R and Galinkin JL (2018) Naloxone dosage for opioid reversal: current evidence and clinical implications. Ther Adv Drug Saf 9:63-88.

Sharma A, Kelly SM, Mitchell SG, Gryczynski J, O'Grady KE, and Schwartz RP (2017) Update on barriers to pharmacotherapy for opioid use disorders. Curr Psychiatry Rep 19:35.

Smith K, Garman L, Wrammert J, Zheng N-Y, Capra JD, Ahmed R, and Wilson PC (2009) Rapid generation of fully human monoclonal antibodies specific to a vaccinating antigen. Nat Protoc 4:372-384.

Smith LC, Bremer PT, Hwang CS, Zhou B, Ellis B, Hixon MS, and Janda KD (2019) Monoclonal antibodies for combating synthetic opioid intoxication. J Am Chem Soc 141:10489-10503.

Spanier JA, Frederick DR, Taylor JJ, Heffernan JR, Kotov DI, Martinov T, Osum KC Ruggiero JL, Rust BJ, Landry SJ, et al. (2016) Efficient generation of monoclonal antibodies against peptide in the context of MHCII using magnetic enrichment. Nat Commun 7:11804

Starkie DO, Compson JE, Rapecki S, and Lightwood DJ (2016) Generation of recombinant monoclonal antibodies from immunised mice and rabbits via flow cytometry and sorting of antigen-specific IgG+ memory B cells. PLoS One 11: e0152282.

Stevens MW, Henry RL, Owens SM, Schutz R, and Gentry WB (2014) First human study of a chimeric anti-methamphetamine monoclonal antibody in healthy volunteers. MAbs 6:1649-1656.

Sulima A, Jalah R, Antoline JFG, Torres OB, Imler GH, Deschamps JR, Beck Z Alving CR, Jacobson AE, Rice KC, et al. (2018) A stable heroin analogue that can serve as a vaccine hapten to induce antibodies that block the effects of heroin and its metabolites in rodents and that cross-react immunologically with related drugs of abuse. J Med Chem 61:329-343.

Taylor JJ, Laudenbach M, Tucker AM, Jenkins MK, and Pravetoni M (2014) Haptenspecific naïve B cells are biomarkers of vaccine efficacy against drugs of abuse J Immunol Methods 405:74-86.

Tenney RD, Blake S, Bremer PT, Zhou B, Hwang CS, Poklis JL, Janda KD, and Banks ML (2019) Vaccine blunts fentanyl potency in male rhesus monkeys. Neuropharmacology 158:107730.

Address correspondence to: Marco Pravetoni, Department of Pharmacology, University of Minnesota, Nils Hasselmo Hall, 312 Church St SE, Minneapolis, MN 55455. E-mail: prave001@umn.edu 